\section{Necrotizing fasciitis of the lower limbs}

\author{
Paola Muggeo,1 Giampaolo Arcamone,2 \\ Antonino Rizzo, ${ }^{1}$ Nicola Santoro 1 \\ 1U.O. Pediatria F. Vecchio \\ Oncoematologia Pediatrica; \\ 2U.O. Chirurgia Pediatrica, A. \\ Ospedaliero-Universitaria Policlinico, \\ Bari, Italy
}

\begin{abstract}
We report an uncommon severe soft-tissue infection of the thighs in a male child with acute lymphoblastic leukemia. Early and aggressive medical treatment and the conservative surgical approach were successful. Necrotizing fasciitis should be suspected in any soft-tissue infection until it can be definitely ruled out, since prompt deliver of medical and surgical intervention is essential.
\end{abstract}

\section{Introduction}

Necrotizing fasciitis (NF) is an uncommon life-threatening infection of the soft tissue compartment (skin, subcutaneous soft tissue, superficial and deep fascia, muscle) requiring urgent surgical and medical treatment. Early diagnosis and subsequent speedy treatment are essential for improving outcomes. Necrotizing fasciitis may result from any insult to the integumentary system (skin biopsy, needle puncture, laceration, insect bite), more commonly in patients with chronic debilitating diseases, such as diabetes mellitus, chronic hepatitis, chronic renal failure, cancer, HIV.1,2

Necrotizing fasciitis can be divided into two categories depending on the causative agent. Type I disease is polymicrobial, caused by a mixture of aerobic, facultative or anaerobic bacteria (Klebsiella sp, Vibrio sp., Aeromonas, Staphylococcus, Clostridia) type II disease is usually monomicrobial, caused by Group A Streptococci. In general, group II NF is a rapid and progressive disease with a poorer prognosis than type I.2,3

Staphylococcus aureus is emerging as a causative agent for NF. Necrotizing fasciitis associated with Staphylococcus aureus has been reported to be less virulent, with subacute onset and lower mortality rate as compared to similar infections caused by other organisms. $4,5,6,7$

Early treatment of NF is crucial for survival. Broad-spectrum aggressive antibiotics, together with adequate support to maintain vital function should be promptly warranted. Surgical debridement or incisional drainage, repeated if necessary, is essential for the best management of NF. Intravenous immunoglobulin infusion has also shown to reduce mortality in NF, particularly if associated with group A Streptococcal infection.1,2

\section{Case Report}

We report on a 7-year old male child suffering from acute lymphoblastic leukemia. In the intensive phase of treatment (reinduction of AIEOP ALL 2006 protocol), the child presented with pain in both thighs. Intramuscular asparaginase had been administered two days earlier. A few hours later, he developed a high fever and malaise. He was empirically treated with ceftazidime and amikacine. In a few hours, erythematous round flat lesions appeared on the right thigh, and soon after both lower limbs enlarged and became eythematous, with tense edema and bullae. His general conditions worsened and he developed tachycardia, hypotension and shock. Teicoplanine was administered while ceftazidime was changed to meropenem and gamma-globulin were infused. Aggressive fluid resuscitation and vasoactive amine were also necessary. Laboratory tests showed elevated serum Creactive protein (CRP) $(288 \mathrm{mg} / \mathrm{L})$ and creatinine kinase (CK) (702 U/L) with low white blood cell count (WBC) $(0.45 \times 109 / \mathrm{L})$, due to aplasia subsequent to chemotherapy. Blood cultivation detected a Staphylococcus aureus. MRI revealed extensive high intensity signal in subcutaneous tissue, superficial fascia, muscles and deep fascia of the right thigh. Imaging of the controlateral thigh revealed similar but less severe results. Bilateral surgical debridement and drainage was performed. The child was discharged 30 days after in good clinical conditions. He completed the treatment for leukemia.

\section{Discusssion}

Necrotizing fasciitis is a rare but life-threatening clinical entity, sometimes reported in children. Since early diagnosis and speedy treatment are essential for improving outcomes and reducing the mortality rate, a high index of suspicion should be maintained In our patient, already admitted to the hospital for chemotherapy, empirical broad spectrum antibiotic treatment was promptly started, and NF was suspected very early. This probably allowed a less invasive necrotizing component in a child who was heavily immunocompromised and, therefore, at higher risk for severe
Correspondence: Paola Muggeo, U.0. Pediatria F. Vecchio, Oncoematologia Pediatrica, A. Ospedaliero-Universitaria Policlinico, Bari, Italy. E-mail: paola.muggeo@tiscali.it

Key words: necrotizing fasciitis, lymphoblastic leukemia, lower limbs.

Received for publication: 24 October 2011. Accepted for publication: 24 October 2011.

This work is licensed under a Creative Commons Attribution NonCommercial 3.0 License (CC BYNC 3.0).

(C) Copyright P. Muggeo et al., 2012

Licensee PAGEPress, Italy

Pediatric Reports 2012; 4:e4

doi:10.4081/pr.2012.e4

infections and fulminant NF

We should also consider that, according to previous reports, Staphylococcus aureus as causative agent of NF is associated with a subacute and less virulent disease.5,6 It may also be that in our patient this etiology contributed to a less devastating outcome. In fact, a nonmutilating surgical approach together with early medical treatment allowed optimal management of NF.

One of the most challenging aspects of NF is its initial similarities with cellulitis. ${ }^{2}$ As reported, cellulitis improves with wide spectrum therapy and at onset rarely presents with severe malaise and a clinical picture of septic shock. Moreover, CT and MRI involvement of deep and superficial fascia is typical for NF.1,8 Due to the severity of the disease, and the importance of early treatment, NF should be suspected in any subcutaneous infections until it can be definitely ruled out.

Staphylococcus aureus is increasingly emerging as causative agent of NF. Our experience confirms previous reports that NF associated with Staphylococci represents a less virulent clinical entity. In our opinion, NF caused by Staphylococcus aureus might be successfully cured by early medical treatment with a more conservative surgical approach.

\section{References}

1. Anaya DA and Dellinger EP. Nectrotizing soft-tissue infection: diagnosis and management. Clin Infect Dis 2007;44:705-10.

2. Cheung JPY, Fung B, Tang WM, Ip WY. A review of necrotizing fasciitis in the extremities. Hong Kong Med J 2009;15:4452.

3. Iwata Y, Sato S, Murase Y, et al. Five cases of necrotizing fasciitis: lack of skin inflam- 
matory signs as a clinical clue for the fulminant type. J Dermatol 2008;35:719-25.

4. Miller LG, Perdreau-Remington F, Rieg G, et al. Necrotizing fasciitis caused by community-associated methicillin-resistant Staphylococcus aureus in Los Angeles. N Engl J Med 2005;352:1445-53.
5. Wong $\mathrm{CH}$, Tan SH. Subacute necrotizing fasciitis. Lancet 2004;364:1376.

6. Saliba WR, Goldstein LH, Raz R, et al. Subacute necrotizing fasciitis caused by gas-producing Staphylococcus aureus. Eur J Clin Microbiol Infect Dis 2003;22:612-14.

7. Wong $\mathrm{CH}$, Wang YS. What is subacute necrotizing fasciitis? A proposed clinical diagnostic criteria. J Infect 2006;52:415-9.

8. Struk DW, Munk PL, Lee MJ, et al. Imaging of soft tissue infections. Radiol Clin North Am 2001;39:277-303. 